\title{
Cambios de la fracción hidrosoluble de huevo de gallinas alimentadas con harina de camarón almacenado a diferentes tiempos y temperaturas
}

\section{Changes in the water soluble fraction of eggs from hens fed with shrimp meal stored at different times and temperatures}

\author{
María E. Carranco-Jáureguii, Silvia Carrillo-Domíngueza , Benjamín Fuente-Martínez ${ }^{\mathrm{b}}$, Ernesto Ávila- \\ González ${ }^{\mathrm{b}}$, María de Lourdes Solano ${ }^{\mathrm{a}}$
}

\begin{abstract}
RESUMEN
El objetivo de este estudio fue determinar el efecto de las condiciones de almacenamiento, sobre la fracción hidrosoluble del huevo de gallinas alimentadas con una dieta convencional y una dieta con $20 \%$ de harina de cabezas de camarón (HCC). Noventa (90) gallinas I sa-Brown de 32 semanas de edad se distribuyeron en dos tratamientos: Testigo y HCC. Durante cuatro semanas de ensayo se evaluaron las variables productivas. Los huevos recolectados $(n=250)$ para este estudio, se almacenaron por 15 y 30 días, a $20^{\circ}$ y $4^{\circ} \mathrm{C}$, tomando como referencia huevos recién puestos (sin almacenar). Los parámetros estudiados fueron peso de huevo, Unidades Haugh, pH, proteína, aminoácidos indispensables y vitaminas hidrosolubles. Los resultados se analizaron utilizando un diseño factorial $2 \times 2 \times 3$ y las diferencias entre medias se compararon mediante la prueba de Tukey. Los resultados obtenidos mostraron que los índices de calidad de la fracción hidrosoluble de huevo se vieron afectadas significativamente $(P<0.05)$ por el tiempo y la temperatura de almacenamiento, y diferencias $(P<0.05)$ solo en proteína por el efecto de la inclusión de HCC. A temperatura ambiente $\left(20^{\circ} \mathrm{C}\right)$ y mayor tiempo de almacenamiento (30 días), provocó una disminución en la calidad interna del huevo de acuerdo a los resultados de este estudio. Por lo tanto, se concluye que tanto la temperatura como el tiempo son uno de los principales factores que llegan a influir en la calidad de la fracción hidrosoluble del huevo durante el almacenamiento.
\end{abstract}

PALABRAS CLAVE: Harina, Camarón, Calidad de huevo, Almacenamiento.

\begin{abstract}
The objective of this study was to determine the effect of storage conditions on the water soluble fraction in eggs from hens fed with a conventional diet and a $20 \%$ shrimp by-products meal (SBPM). Ninety (90) I sa-Brown laying hens 32 wk age were distributed in two treatments: Control and SBPM. The productive variables were evaluated during 4 wk. Eggs ( $n=250)$ collected for this study, were stored at 15 and $30 \mathrm{~d} / 20^{\circ}$ and $4{ }^{\circ} \mathrm{C}$, taking as reference fresh eggs (not stored). The parameters studied were egg weight, Haugh Units, $\mathbf{p H}$, crude protein, essential amino acids and water soluble vitamins. The results were analyzed using a $2 \times 2 \times 3$ factorial design and the differences between means were compared using the Tukey test. The data obtained showed that the quality indexes of the water soluble fraction of egg were significantly affected $(P<0.05)$ by the time and the temperature of storage and differences $(P<0.05)$ in protein alone due to the effect of SBPM inclusion. At room temperature $\left(20^{\circ} \mathrm{C}\right)$ and longer storage time $(30$ d), a decrease in internal egg quality was observed according to the results of this study. Therefore, it is concluded that both temperature and time are one of the main factors influencing the eggs quality of the water-soluble fraction of the egg during storage.
\end{abstract}

KEY WORDS: Shrimp heads meal, Egg quality, Storage.

Recibido el 11 de mayo de 2016. Aceptado el 25 de abril de 2017.

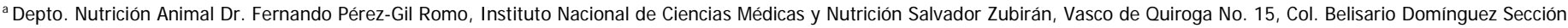
XVI, Ciudad de México. México.

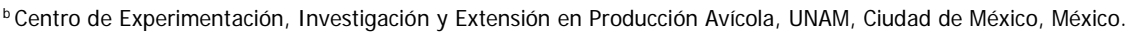

*Autor para correspondencia: rexprimero@hotmail.com 


\section{INTRODUCCIÓN}

En México las cabezas de camarón (Litopenaeus spp) son un subproducto natural que no tiene utilidad a nivel industrial, ya que se desperdician alrededor del $33 \%$, por lo que se considera un contaminante. Desde el año 1990 a la fecha, se han buscado alternativas para el uso de este subproducto de la industria pesquera, para utilizarse como fuente de proteína animal en dietas acuícolas $^{(1-3)}$. Por las características de la harina de cabezas de camarón (HCC), en cuanto a su contenido de proteína ( 45 a $55 \%$ ), se ha utilizado en la avicultura. Los huevos provenientes de aves alimentadas con esta harina han mostrado un ligero incremento de los componentes de la parte hidrosoluble del huevo, en comparación con los huevos provenientes de una dieta convencional ${ }^{(4,5)}$.

La Unión Nacional de Avicultores ${ }^{(6)}$ reporta un consumo de huevo en México per capita anual de 22 kg (305 huevos/persona/año aproximadamente). Siendo el huevo un alimento completo, de bajo costo y versátil en su preparación, éste no llega al consumidor, fresco, es decir, recién puesto por la gallina, sino que se almacena a tiempos y temperaturas diversas. La Norma Oficial Mexicana ${ }^{(7)}$ define $a$ un huevo fresco aquel que no excede los 15 días después de la puesta. Durante este periodo la cámara de aire se incrementa debido a la pérdida de agua y se presenta una modificación en el pH, siendo los principales factores la temperatura, el tiempo y la humedad relativa los que afectan la calidad del huevo durante el almacenamiento ${ }^{(8)}$. Este cambio, más el efecto de los compuestos provenientes de nuevas materias primas que se adicionen a la dieta de aves, pueden repercutir en la calidad física y química del huevo ${ }^{(9,10)}$. De acuerdo con los resultados publicados por otros autores ${ }^{(4)}$ quienes trabajaron con HCC en niveles de inclusión de $10,15,20$ y $25 \%$, indican que el mejor nivel fue el del $20 \%$, reportando un huevo de buena calidad. Niveles superiores provocaron una modificación en las características sensoriales (olor y sabor) del huevo, por lo cual se consideró adecuado trabajar con $20 \%$ de inclusión, para determinar si las propiedades físicas y composición química de la parte hidrosoluble del huevo proveniente de gallinas alimentadas con una dieta convencional y la dieta propuesta por los autores mencionados ${ }^{(4)}$ sufren modificaciones durante condiciones estandarizadas de almacenamiento. Por lo tanto el objetivo de esta investigación fue determinar los cambios físicos y químicos de la fracción hidrosoluble, de huevos provenientes de gallinas cuya dieta incluyó $20 \%$ de harina de cabezas de camarón, almacenados a 0,15 y 30 días $/ 4^{\circ}$ y $20^{\circ} \mathrm{C}$.

\section{MATERIAL Y MÉTODOS}

El estudio se realizó en el Departamento de Nutrición Animal Dr. Fernando Pérez-Gil Romo, del Instituto Nacional de Ciencias Médicas y Nutrición Salvador Zubirán y en el CEIEPAv (Centro de Enseñanza, Investigación y Extensión en Producción Avícola) de la Facultad de Medicina Veterinaria y Zootecnia (UNAM). Se utilizaron $30 \mathrm{~kg}$ de harina de cabezas de camarón (HCC) procedentes de Proteínas Marinas y Agropecuarias S.A. de C.V. (Guadalajara, Jalisco, México). La harina de HCC se guardó en bolsas de plástico negras y se congelaron a $-20^{\circ} \mathrm{C}$ hasta su utilización. A esta harina se le realizaron los siguientes análisis de acuerdo con las técnicas estandarizadas publicadas por $A O A C^{(11)}$ : proteína cruda (Método 976.05), cenizas (Método 942.05), extracto etéreo (Método 920.39), minerales (Métodos Cap. 4-40), perfil de aminoácidos (HPLC) (Manual Waters Acc-QTAG, Manual No. WAT052874, abril 1993) y energía bruta por bomba calorimétrica Parr.

\section{Preparación de dietas y ensayo con aves}

Las dietas se formularon por medio del programa NUTRION 5 Básico/Nutrion 5 PRO, sistema computarizado para la formulación de alimentos balanceados (Guadalajara, Jal. México) Nutrion Windows ${ }^{\mathrm{TM}}$ (Versión 5.0 Pro), cubriendo las necesidades nutrimentales establecidas por el National Research Council (NRC)(12) para gallinas ponedoras, y con base en los aminoácidos totales.

El procedimiento utilizado en este estudio fue aprobado por el Subcomité Institucional para el Cuidado y uso de Animales Experimentales (SICUAE), Facultad de Medicina Veterinaria y Zootecnia de la Universidad Nacional Autónoma de México(13). Noventa (90) gallinas Isa-Brown de 32 semanas de edad se distribuyeron al azar en dos 
tratamientos de 45 gallinas cada uno y con cinco repeticiones: $\mathrm{T} 1=$ Testigo y $\mathrm{T} 2=20 \% \mathrm{HCC}$. El agua y el alimento se proporcionaron a libre acceso por cuatro semanas que duró el experimento. Durante este tiempo se midieron las variables productivas (peso y masa del huevo, consumo de alimento y conversión alimenticia). Al finalizar las cuatro semanas, se recolectaron 250 huevos/tratamiento y se almacenaron de la siguiente manera: día 0 ( 50 huevos); 15 días $/ 20^{\circ} \mathrm{C}$ (50 huevos) y 15 días $/ 4{ }^{\circ} \mathrm{C}$ (50 huevos); 30 días $/ 20^{\circ} \mathrm{C}$ (50 huevos) y 30 días $/ 4$ ${ }^{\circ} \mathrm{C}$ (50 huevos).

Experimento 1. A los huevos frescos y almacenados 15 y $30 \mathrm{~d} / 20$ y $4{ }^{\circ} \mathrm{C}$ se les determinó peso de huevo y Unidades Haugh (UH), utilizando un equipo automatizado para estas mediciones (Technical Service and Supplies, Inc., England, UK). Este sistema consta de un microprocesador (QCM+) conectado a una balanza digital y un micrómetro para la medición de altura de albúmina. El QCM+ recopila los datos de los instrumentos en línea y muestra una lectura, la cual es transferida a una computadora equipada con el software de calidad de huevo. Las UH se calcularon a partir de la altura de albúmina y peso de huevo por el mismo procesador.

Experimento 2. Las muestras de huevo del Experimento 1, conforme fueron cumpliendo las condiciones de tiempo de almacenamiento, se mezclaron (yema y albúmina), se midió el pH utilizando un potenciómetro HANNA Instrument pH210, Microprocesor $\mathrm{pH}$ Meter y posteriormente se liofilizaron. Al huevo liofilizado se le realizaron los análisis de proteína cruda, vitaminas hidrosolubles y aminoácidos indispensables.

\section{Análisis estadístico}

Los resultados de las variables productivas se analizaron mediante un diseño completamente al azar (ANOVA) y las diferencias entre medias por la prueba de Tukey con un nivel de confianza del $95 \%$, con el programa SPSS versión 11.0 para Windows (SPSS Inc, Chicago IL, USA). El modelo utilizado fue:

$$
Y i j=\mu+T i+\epsilon i j
$$

Donde $Y i j=$ variable de respuesta; $\mu=$ media general; $\mathrm{Ti}=$ efecto del i-esimo tratamiento; $\epsilon \mathrm{ij}=$ error experimental.
Los resultados de los análisis físicos y químicos de la fracción hidrosoluble del huevo se analizaron de acuerdo a un diseño factorial $2 \times 2 \times 3$ siendo los factores: tratamiento, tiempo y temperatura. Para la comparación entre medias se utilizó la prueba Tukey con un nivel de confianza del 95\%, SPSS versión 11.0 para Windows (SPSS Inc, Chicago IL, USA). El modelo utilizado fue

$$
\begin{aligned}
& \quad Y i j k=\mu+a i+\beta j+\gamma_{k}(a \beta) i j+(a v)_{i k}+(\beta \gamma)_{j k} \\
& +(a \beta y) i j k+\epsilon i j k(I)
\end{aligned}
$$

Donde Yijk= variable de respuesta; $\mu=$ media; $a i=$ efecto del $\mathrm{i}$-esimo tratamiento; $\beta \mathrm{j}=$ efecto del j-esimo tiempo; $v_{k}=$ efecto del k-esimo temperatura; $(a \beta) i j=$ efecto de la interacción del tratamiento y tiempo; $(\beta \vee)_{\mathrm{jk}}=$ efecto de la interacción del tiempo y la temperatura; $(a \beta \vee) i j k=$ interacción del

Cuadro 1. Composición química de la harina de cabezas de camarón*

\begin{tabular}{lr}
\hline Humedad, $\mathrm{g} / 100 \mathrm{~g}$ & $9.02 \pm 0.01$ \\
Cenizas, $\mathrm{g} / 100$ & $32.82 \pm 0.03$ \\
Extracto etéreo, g/100 g & $0.96 \pm 0.03$ \\
Proteína cruda, g/100 g & $39.64 \pm 0.26$ \\
Carbohidratos totales, $\mathrm{g} / 100 \mathrm{~g}$ & 26.58 \\
Energía bruta, Kjoules/g & $10.19 \pm 0.37$ \\
Calcio, $\mathrm{mg} / 100 \mathrm{~g}$ & $4.58 \pm 0.15$ \\
Sodio, $\mathrm{mg} / 100 \mathrm{~g}$ & $10.45 \pm 0.28$ \\
Magnesio, mg/100 g & $14.02 \pm 0.21$ \\
\hline Aminoácidos & \\
Isoleucina & \\
Leucina & $4.12 \pm 0.02$ \\
Lisina & $6.85 \pm 0.02$ \\
Metionina & $10.12 \pm 0.03$ \\
Cistina & $1.58 \pm 0.02$ \\
Fenilalanina & $2.02 \pm 0.01$ \\
Tirosina & $4.35 \pm 0.01$ \\
Alanina & $4.18 \pm 0.01$ \\
Ácido aspártico & $6.55 \pm 0.01$ \\
Glutamina & $9.25 \pm 0.02$ \\
Treonina & $14.95 \pm 0.01$ \\
Glicina & $3.85 \pm 0.03$ \\
Prolina & $7.81 \pm 0.02$ \\
Serina & $5.25 \pm 0.02$ \\
Valina & $3.46 \pm 0.01$ \\
Arginina & $5.36 \pm 0.03$ \\
Histidina & $3.75 \pm 0.01$ \\
\hline
\end{tabular}

*Se reporta la media y desviación estándar de 6 repeticiones.

** (g/100 g de proteína). 
tratamiento, el tiempo y la temperatura; $\epsilon i j k(I)=$ error experimental.

\section{RESULTADOS}

\section{Composición química de la harina de cabezas de camarón (HCC)}

En el Cuadro 1 se presentan los resultados del análisis químico aproximado, siendo las fracciones más abundantes proteína cruda (PC) (39.64\%), cenizas (32.82 \%), Mg (14.02 mg/100 g) y Na $(10.45 \mathrm{mg} / 100 \mathrm{~g})$. Con base a la composición química y de aminoácidos de la harina se formularon las dietas experimentales (Cuadro 2).

\section{Variables productivas}

Al analizar los promedios, se encontró que no hay una diferencia significativa $(P>0.05)$ para las variables productivas entre los tratamientos, indicando que la inclusión de HCC no causó problemas. Carranco et $a\left(^{(4)}\right.$ mencionan que las inclusiones mayores al $20 \%$ de HCC presentaron diferencias $(P<0.05)$ en consumo de alimento, posiblemente debido al contenido de sal o que hayan detectado algún olor o sabor desagradable (pescado) (Cuadro 3).

\section{Perfil de aminoácidos}

No se presentaron diferencias estadísticas $(P>0.05)$ en los resultados de aminoácidos tanto en huevo testigo como con HCC, así como en tiempos ni temperaturas (Cuadro 4).

Peso de huevo, proteína cruda (PC), pH y unidades Haugh (UH)

En el Cuadro 5 se presentan los resultados de las respuestas entre la adición de HCC, temperatura y tiempo, encontrándose diferencias en peso de huevo a $4^{\circ} \mathrm{C} / 15$ días, para PC sólo se presentaron
Cuadro 2. Dietas experimentales suplementadas con harina de cabezas de camarón

\begin{tabular}{|c|c|c|}
\hline Ingredientes (g/kg) & Testigo & $\begin{array}{l}\text { Harina de cabezas } \\
\text { de camarón }\end{array}$ \\
\hline Sorgo & 673.957 & 648.857 \\
\hline Harina de cabezas de camarón & 0.000 & 200.000 \\
\hline Carbonato de calcio & 107.159 & 82.920 \\
\hline Pasta de soya & 182.800 & 37.371 \\
\hline Fosfato de calcio 1821 & 11.931 & 13.233 \\
\hline Aceite vegetal & 10.000 & 9.770 \\
\hline Sal & 3.626 & 3.048 \\
\hline Premezcla de vitaminasa & 2.500 & 2.500 \\
\hline L-Lisina $\mathrm{HCl}$ & 2.426 & 0.000 \\
\hline DL-Metionina & 2.249 & 0.000 \\
\hline Secuestrante de micotoxinas & 1.000 & 1.000 \\
\hline L-Treonina & 0.703 & 0.000 \\
\hline Pigmento amarillob & 0.500 & 0.500 \\
\hline Cloruro de colina 60\% & 0.500 & 0.500 \\
\hline Bacitracina zinc & 0.300 & 0.300 \\
\hline Pigmento rojoc & 0.200 & 0.000 \\
\hline Antioxidante & 0.150 & 0.000 \\
\hline Total & 1000 & 1000 \\
\hline \multicolumn{3}{|c|}{ Análisis calculado } \\
\hline Energía metabolizable, kcal/kg & 2750 & 2750 \\
\hline Proteína cruda, \% & 15.00 & 15.10 \\
\hline Metionina, \% & 0.456 & 0.495 \\
\hline Metionina + cistina, \% & 0.690 & 0.984 \\
\hline Calcio total, $\%$ & 4.178 & 3.450 \\
\hline Fósforo disponible, $\%$ & 0.340 & 0.340 \\
\hline Sodio, $\%$ & 0.150 & 0.150 \\
\hline Lisina, \% & 0.860 & 2.268 \\
\hline Treonina, \% & 0.620 & 1.041 \\
\hline Triptófano, \% & 0.191 & 0.177 \\
\hline \multicolumn{3}{|c|}{$\begin{array}{l}\text { a Vitamina A, } 12,000 \mathrm{UI} ; \mathrm{D}_{3} 2,500 \mathrm{UIP} ; \mathrm{E}, 30 \mathrm{UI} ; \mathrm{K}, 2 \mathrm{mg} ; \mathrm{B}_{1}, 2.25 \mathrm{mg} ; \mathrm{B}_{2}, 7.5 \mathrm{mg} ; \\
\mathrm{B}_{6}, 3.4 \mathrm{mg} ; \mathrm{B}_{12}, 0.02 \mathrm{mg} ; \text { ácido D-pantoténico, } 12.5 \mathrm{mg} ; \text { biotina, } 0.125 \mathrm{mg} \text {; ácido } \\
\text { fólico, } 1.5 \mathrm{mg} \text {. }\end{array}$} \\
\hline \multicolumn{3}{|c|}{ b Xantofilas amarillas de la flor de Cempazuchil (15 ppm). } \\
\hline
\end{tabular}

Cuadro 3. Variables productivas de gallinas alimentadas con harina de cabezas de camarón (HCC)

\begin{tabular}{lcccc}
\hline & Producción huevo (\%) & Peso huevo (g) & Conversión alimenticia & Consumo alimento ave/día/g \\
\hline Testigo & $88.35 \pm 6.26$ & $64.20 \pm 1.30$ & $2.07 \pm 0.11$ & $118 \pm 2.54$ \\
HCC & $83.02 \pm 7.78$ & $64.24 \pm 2.12$ & $2.11 \pm 0.14$ & $112.8 \pm 5.56$ \\
\hline$(P>0.05)$. & & & &
\end{tabular}


Cuadro 4. Promedios de la composición de aminoácidos esenciales ( $\mathrm{g} / 100 \mathrm{~g}$ de proteína) en huevo almacenados a diferentes tiempos y temperaturas

\begin{tabular}{ccccccccccc}
\hline & Días & \multicolumn{1}{c}{ Isoleucina } & \multicolumn{1}{c}{ Leucina } & \multicolumn{1}{c}{ Lisina } & \multicolumn{1}{c}{ Metionina } & \multicolumn{1}{c}{ Fenilalanina } & \multicolumn{1}{c}{ Treonina } & Valina & Arginina & Histidina \\
\hline \multirow{2}{*}{$20^{\circ} \mathrm{C}$} & 0 & $20.23 \pm 0.15$ & $32.55 \pm 0.007$ & $24.56 \pm 0.02$ & $12.54 \pm 0.007$ & $21.54 \pm 0.00$ & $16.88 \pm 0.00$ & $24.54 \pm 0.01$ & $24.57 \pm 0.01$ & $8.87 \pm 0.007$ \\
& 15 & $19.86 \pm 0.021$ & $31.93 \pm 0.021$ & $23.86 \pm 0.021$ & $12.43 \pm 0.021$ & $21.13 \pm 0.021$ & $16.72 \pm 0.014$ & $23.97 \pm 0.028$ & $24.32 \pm 0.014$ & $8.76 \pm 0.014$ \\
& 30 & $19.82 \pm 0.014$ & $31.18 \pm 0.014$ & $23.52 \pm 0.021$ & $12.36 \pm 0.021$ & $21.03 \pm 0.014$ & $16.66 \pm 0.014$ & $23.76 \pm 0.014$ & $24.03 \pm 0.028$ & $8.63 \pm 0.021$ \\
$4^{\circ} \mathrm{C}$ & 0 & $20.23 \pm 0.15$ & $32.55 \pm 0.007$ & $24.56 \pm 0.02$ & $12.54 \pm 0.007$ & $21.54 \pm 0.00$ & $16.88 \pm 0.00$ & $24.54 \pm 0.01$ & $24.57 \pm 0.01$ & $8.87 \pm 0.007$ \\
& 15 & $20.04 \pm 0.014$ & $32.46 \pm 0.014$ & $24.26 \pm 0.021$ & $12.48 \pm 0.021$ & $21.22 \pm 0.014$ & $16.77 \pm 0.007$ & $24.51 \pm 0.014$ & $24.48 \pm 0.00$ & $8.78 \pm 0.014$ \\
& 30 & $19.96 \pm 0.021$ & $32.16 \pm 0.014$ & $24.22 \pm 0.035$ & $12.43 \pm 0.028$ & $21.19 \pm 0.028$ & $16.73 \pm 0.028$ & $24.26 \pm 0.014$ & $24.36 \pm 0.021$ & $8.76 \pm 0.014$ \\
& & & & & Harina de camarón & & \\
$20^{\circ} \mathrm{C}$ & 0 & $21.42 \pm 0.02$ & $33.23 \pm 0.02$ & $25.86 \pm 0.02$ & $13.83 \pm 0.01$ & $22.05 \pm 0.01$ & $17.25 \pm 0.01$ & $25.66 \pm 0.02$ & $25.88 \pm 0.007$ & $9.94 \pm 0.01$ \\
& 15 & $21.12 \pm 0.021$ & $32.46 \pm 0.021$ & $25.20 \pm 0.014$ & $13.44 \pm 0.01$ & $21.92 \pm 0.021$ & $16.58 \pm 0.038$ & $25.28 \pm 0.014$ & $25.60 \pm 0.014$ & $9.71 \pm 0.007$ \\
& 30 & $19.96 \pm 0.021$ & $31.99 \pm 0.021$ & $25.06 \pm 0.021$ & $13.24 \pm 0.042$ & $24.56 \pm 0.021$ & $16.53 \pm 0.028$ & $25.14 \pm 0.028$ & $25.36 \pm 0.028$ & $9.36 \pm 0.021$ \\
$4^{\circ} \mathrm{C}$ & 0 & $21.42 \pm 0.02$ & $33.23 \pm 0.02$ & $25.86 \pm 0.02$ & $13.83 \pm 0.01$ & $22.05 \pm 0.01$ & $17.25 \pm 0.01$ & $25.66 \pm 0.02$ & $25.88 \pm 0.007$ & $9.94 \pm 0.01$ \\
& 15 & $21.23 \pm 0.028$ & $32.93 \pm 0.021$ & $25.53 \pm 0.028$ & $13.56 \pm 0.021$ & $21.96 \pm 0.021$ & $17.14 \pm 0.028$ & $25.46 \pm 0.028$ & $25.79 \pm 0.021$ & $9.86 \pm 0.021$ \\
& 30 & $21.20 \pm 0.014$ & $32.84 \pm 0.014$ & $25.42 \pm 0.021$ & $13.46 \pm 0.021$ & $21.96 \pm 0.014$ & $17.02 \pm 0.021$ & $25.10 \pm 0.021$ & $25.78 \pm 0.014$ & $9.76 \pm 0.021$ \\
\hline
\end{tabular}

$(P>0.05)$.

Cuadro 5. Respuesta de la adición de harina de cabezas de camarón la temperatura y el almacenamiento de huevo sobre el peso, proteína cruda, pH y Unidades Haugh

\begin{tabular}{|c|c|c|c|c|c|}
\hline \multirow[b]{2}{*}{ Dieta } & \multicolumn{2}{|c|}{$\begin{array}{c}\text { Testigo } \\
\text { Temperatura }\end{array}$} & \multicolumn{3}{|c|}{$\begin{array}{l}\text { Harina de camarón } \\
\text { Temperatura }\end{array}$} \\
\hline & $20^{\circ} \mathrm{C}$ & $4^{\circ} \mathrm{C}$ & $20^{\circ} \mathrm{C}$ & $4{ }^{\circ} \mathrm{C}$ & Promedio \\
\hline \multicolumn{6}{|c|}{ Peso huevo $(\mathrm{g})$} \\
\hline \multicolumn{6}{|l|}{ Días de almacenamiento } \\
\hline 0 & 64.16 & 64.49 & 64.28 & 64.28 & $64.03^{a}$ \\
\hline 15 & 62.77 & 60.10 & 61.35 & 59.96 & $61.05^{b}$ \\
\hline 30 & 63.87 & 63.87 & 64.24 & 63.23 & $63.80^{\mathrm{a}}$ \\
\hline Tiempo $\times$ Temp & $63.60^{\mathrm{a}}$ & $62.82^{b}$ & $63.29^{a}$ & $62.49 \mathrm{~b}$ & \\
\hline \multicolumn{6}{|c|}{ Proteína cruda (\%) } \\
\hline 0 & 48.8 & 48.8 & 50.9 & 50.0 & $49.6^{\mathrm{a}}$ \\
\hline 15 & 50.0 & 50.3 & 49.7 & 50.6 & $50.1^{b}$ \\
\hline 30 & 47.3 & 50.1 & 48.6 & 50.2 & $49.0^{a}$ \\
\hline Tiempo $\times$ Temp & $48.7^{a}$ & $49.7^{a}$ & $49.7^{a}$ & $50.3^{a}$ & \\
\hline \multicolumn{6}{|c|}{$\mathrm{pH}$} \\
\hline 0 & 7.35 & 7.35 & 7.50 & 7.50 & $7.42^{\mathrm{c}}$ \\
\hline 15 & 8.70 & 8.85 & 8.70 & 8.85 & $8.77^{b}$ \\
\hline 30 & 9.25 & 9.40 & 9.25 & 9.40 & $9.32^{\mathrm{a}}$ \\
\hline Tiempo × Temp & $8.43^{a}$ & $8.53^{a}$ & $8.48^{a}$ & $8.58^{a}$ & \\
\hline \multicolumn{6}{|c|}{ Unidades Haugh } \\
\hline 0 & 76.14 & 76.14 & 78.32 & 78.32 & $77.23^{a}$ \\
\hline 15 & 50.84 & 69.57 & 58.90 & 65.24 & $61.14^{b}$ \\
\hline 30 & 41.52 & 64.44 & 43.95 & 65.81 & $53.93^{c}$ \\
\hline $\begin{array}{l}\text { Tiempo } \times \text { Temp } \\
\text { EEM }=1.56\end{array}$ & $56.17^{c}$ & $70.05^{a}$ & $60.39^{b}$ & $69.79^{a}$ & \\
\hline
\end{tabular}

abc Valores con diferente letra en fila o columna son diferentes $(P<0.05)$. Tiempo $\times$ Temp= promedio de días de almacenamiento por temperatura. EEM= error estándar de la media. diferencias entre tiempos de almacenamiento y $\mathrm{UH}$; se encontraron diferencias significativas en tiempo, temperatura y dietas $(P<0.05)$.

El Cuadro 6 muestra el efecto de la temperatura y tiempo sobre el peso, $\mathrm{PC}, \mathrm{pH}$ y UH del huevo. Se reportan diferencias $(P<0.05)$ a 30 días de almacenamiento y las temperaturas para las variables estudiadas; sin embargo se observa que los mejores resultados fueron para el huevo almacenado a $4{ }^{\circ} \mathrm{C}$.

El efecto de la dieta y el tiempo de almacenamiento del huevo sobre peso, $\mathrm{PC}, \mathrm{pH}$ y UH se presentan en el Cuadro 7, observándose que para el peso de huevo y PC hubo diferencias significativas $(P<0.05)$ tanto en días de almacenamiento como en las dietas, teniendo los valores más bajos a los 30 días de almacenamiento; sin embargo aunque se presentó un aumento en el $\mathrm{pH}$, no hubo diferencias significativas, y en UH se presentaron diferencias $(P<0.05)$ sólo entre dietas.

\section{DISCUSIÓN}

En el presente trabajo los valores obtenidos para la composición química de HCC resultaron ser menores a los reportados por Charley ${ }^{(14)}$ para harina de otro crustáceo (cangrejo $47.2 \%$ ) y por Castro et $a{ }^{(15)}$ quienes informan un contenido de $39.9 \%$ para el crustáceo langostilla. En cuanto a la cantidad 
Cuadro 6. Efecto de la temperatura y el tiempo de almacenamiento del huevo sobre el peso, proteína cruda, pH y unidades Haugh

\begin{tabular}{|c|c|c|c|c|}
\hline \multirow[t]{2}{*}{ Temperatura } & \multicolumn{4}{|c|}{ Días de almacenamiento } \\
\hline & 0 & 15 & 30 & Promedio \\
\hline \multicolumn{5}{|c|}{ Peso huevo (g) } \\
\hline $20^{\circ} \mathrm{C}$ & $64.07^{\mathrm{a}}$ & $63.86^{a}$ & $60.03^{b}$ & $62.65^{b}$ \\
\hline $4^{\circ} \mathrm{C}$ & $64.07^{\mathrm{a}}$ & $64.20^{\mathrm{a}}$ & $62.06^{a}$ & $63.44^{\mathrm{a}}$ \\
\hline \multicolumn{5}{|l|}{$\mathrm{EEM}=0.08$} \\
\hline \multicolumn{5}{|c|}{ Proteína cruda (\%) } \\
\hline $20^{\circ} \mathrm{C}$ & $49.9^{a}$ & $49.8^{a}$ & $48.0^{\mathrm{b}}$ & $49.2^{b}$ \\
\hline $4^{\circ} \mathrm{C}$ & $49.9^{a}$ & $50.4^{\mathrm{a}}$ & $50.1^{\mathrm{a}}$ & $50.2^{\mathrm{a}}$ \\
\hline \multicolumn{5}{|l|}{$\mathrm{EEM}=0.19$} \\
\hline \multicolumn{5}{|c|}{$\mathrm{pH}$} \\
\hline $20^{\circ} \mathrm{C}$ & $7.42^{\mathrm{a}}$ & $8.70^{\mathrm{a}}$ & $9.25^{\mathrm{a}}$ & $8.45^{b}$ \\
\hline $4^{\circ} \mathrm{C}$ & $7.42^{\mathrm{a}}$ & $8.85^{\mathrm{a}}$ & $9.40^{\mathrm{a}}$ & $8.55^{\mathrm{a}}$ \\
\hline \multicolumn{5}{|l|}{$\mathrm{EEM}=0.04$} \\
\hline \multicolumn{5}{|c|}{ Unidades Haugh } \\
\hline $20^{\circ} \mathrm{C}$ & $77.23^{\mathrm{a}}$ & $54.87 \mathrm{c}$ & $42.73^{d}$ & $58.27^{b}$ \\
\hline $4^{\circ} \mathrm{C}$ & $77.23^{\mathrm{a}}$ & $67.41^{b}$ & $65.12^{b}$ & $69.92^{\mathrm{a}}$ \\
\hline $\mathrm{EEM}=1.1$ & & & & \\
\hline
\end{tabular}

abcd Valores con diferente letra en fila o columna son diferentes $(P<0.05)$. $\mathrm{EEM}=$ error estándar de la media.

de cenizas, la HCC mostró un valor similar (32.82 \%) a lo reportado por otros autores que va de 28.9 hasta $33 \%{ }^{(16-18)}$, lo cual se puede explicar por el medio ambiente en el que viven estos crustáceos, ricos en minerales. El extracto etéreo resultó estar presente en muy pequeñas cantidades en HCC ( 0.96 $\%)$, valor similar a lo reportado por Charley(14) de $0.80 \%$ para harina de camarón. Para otros crustáceos se reportan contenidos de cenizas entre 4.9 y $7.29 \%$, explicándose esta variabilidad probablemente a la temporada y sitio geográfico de captura. La energía bruta obtenida en este estudio fue de 10.19 Kjoules/g en HCC, valores similares a los de la harina de carne (11.36 Kjoules/g), que también ha sido empleada como ingrediente en dietas para gallinas ponedoras ${ }^{(18)}$.

Con respecto a los aminoácidos indispensables para las aves, la mayoría se encuentran en cantidades superiores comparada a lo reportado por otros autores ${ }^{(19)}$, a excepción de la arginina (3.72\%) y serina $(2.67 \%$ ) reportada por estos autores, y que en este estudio para HCC arginina fue de $3.75 \%$ y
Cuadro 7. Efecto de la dieta y el tiempo de almacenamiento del huevo sobre el peso, proteína cruda, pH y unidades Haugh

\begin{tabular}{|c|c|c|c|c|}
\hline \multirow[t]{2}{*}{ Dieta } & \multicolumn{4}{|c|}{ Días de almacenamiento } \\
\hline & 0 & 15 & 30 & Promedio \\
\hline \multicolumn{5}{|c|}{ Peso huevo (g) } \\
\hline Testigo & $63.87^{\mathrm{b}}$ & $64.32^{\mathrm{ab}}$ & $61.43^{c}$ & $63.21^{\mathrm{a}}$ \\
\hline Camarón & $64.28^{\mathrm{a}}$ & $63.73^{a b}$ & $60.65^{b c}$ & $62.89^{a}$ \\
\hline \multicolumn{5}{|l|}{$\mathrm{EEM}=0.12$} \\
\hline \multicolumn{5}{|c|}{ Proteína cruda (\%) } \\
\hline Testigo & $48.8^{c}$ & $50.1^{\mathrm{ab}}$ & $48.7^{c}$ & $49.2^{b}$ \\
\hline Camarón & $50.9^{a}$ & $50.2^{\mathrm{ab}}$ & $49.4^{b c}$ & $50.2^{\mathrm{a}}$ \\
\hline \multicolumn{5}{|l|}{$\mathrm{EEM}=0.19$} \\
\hline \multicolumn{5}{|c|}{$\mathrm{pH}$} \\
\hline Testigo & $7.35^{\mathrm{a}}$ & $8.77^{a}$ & $9.32^{\mathrm{a}}$ & $8.48^{a}$ \\
\hline Camarón & $7.50^{\mathrm{a}}$ & $8.77^{a}$ & $9.32^{\mathrm{a}}$ & $8.53^{\mathrm{a}}$ \\
\hline \multicolumn{5}{|l|}{$E E M=0.04$} \\
\hline \multicolumn{5}{|c|}{ Unidades Haugh } \\
\hline Testigo & $76.14^{a}$ & $60.21^{a}$ & $52.98^{a}$ & $63.11^{b}$ \\
\hline Camarón & $78.32^{\mathrm{a}}$ & $62.07^{\mathrm{a}}$ & $54.88^{a}$ & $65.09^{a}$ \\
\hline $\mathrm{EEM}=1.1$ & & & & \\
\hline
\end{tabular}

ab Valores con distinta letra en fila o columna son diferentes $(P<0.05)$. EEM= error estándar de la media.

serina $3.46 \%$. Por su parte Quintero ${ }^{(20)}$, publicó valores mayores para los aminoácidos reportados en este estudio, a excepción de histidina (6.82 \%), valina $(5.36 \%$ ) y lisina (10.12 \%), mayores en este estudio. De acuerdo a la formulación de dietas para las aves ${ }^{(12)}$, sólo fue necesario adicionar a la dieta testigo los aminoácidos metionina, treonina y lisina, a diferencia de las dietas elaboradas con HCC, que fueron buena fuente de lisina ( $10.12 \%$ ) y metionina $(1.58 \%)$.

Como lo menciona Huyghebaert(21), una vez puesto el huevo y durante su almacenamiento, se presentan varios cambios bioquímicos, físicos y mecánicos propios de los constituyentes del huevo. Algunos de estos son el aumento de volumen de la cámara de aire, la licuefacción de la albúmina densa y el debilitamiento de la membrana vitelina que separa la yema y la albúmina ${ }^{(22)}$. A esto, cabe citar que los factores que pueden afectar la calidad interna del huevo son la línea genética, edad de la gallina, así como el tiempo y condiciones de almacenamiento del huevo(23) . 
En peso de huevo se observó una pérdida de éste a los 30 días de almacenamiento en ambas temperaturas. Esto se debe a que el tiempo de almacenamiento va a disminuir el valor de la gravedad específica al ser provocada por la deshidratación interna del huevo. Para conocer esta gravedad específica se llega a aplicar el principio de Arquímides, que menciona que la masa de un cuerpo dividido por su volumen es igual a su peso específico, y que en huevo se reporta entre $1.02 \mathrm{a}$ $1.04^{(24)}$.

El $\mathrm{pH}$ en el huevo recién puesto tiene un valor aproximado de 7.31 y se eleva a 8.53 después de 24 h a $20{ }^{\circ} \mathrm{C}$, alcanzando valores de 9.38 después de 10 días $^{(25)}$. En este trabajo, el pH de huevo fresco fue de 7.35 y 7.50 y fue aumentando conforme pasó el tiempo de almacenamiento tanto en testigo como con HCC a los 15 y 30 d/20 y $4{ }^{\circ} \mathrm{C}$ (8.7-9.25; 8.859.40 y 8.7-9.25; 8.85-9.40 respectivamente), lo cual concuerda con lo señalado por Li-Chan et $a^{(2)}$, quienes encontraron que en huevo fresco (en el momento de la postura) el pH oscilaba entre 7.6 y 8.5. Estos mismos autores encontraron valores de $\mathrm{pH}$ de 9.18 a 3 días de puesta a $3{ }^{\circ} \mathrm{C}$ y después de 21 días un $\mathrm{pH}$ de 9.4 .

El pH del huevo (principalmente de la albúmina) es un factor importante en el control de las propiedades reológicas de geles formados durante el tratamiento térmico $\left(80{ }^{\circ} \mathrm{C}\right)$. Éste depende del equilibrio entre el dióxido de carbono disuelto, iones bicarbonato e iones de carbono que se rigen por la presión parcial del dióxido de carbono del medio ambiente exterior, aumentando las concentraciones de iones bicarbonato a medida que el carbonato disminuye ${ }^{(2)}$. Conforme aumenta el $\mathrm{pH}$ en el huevo durante el almacenamiento, disminuye la elasticidad del gel, la penetración de la fuerza y el índice de viscosidad. Esto traerá como consecuencia que las propiedades funcionales de la albúmina, como gelificación, coagulación, emulsión, formación de espuma, viscosidad y capacidad de retención de agua, se vean afectadas por factores como la fuerza iónica, $\mathrm{pH}$, temperatura, tensión superficial, entre otros, haciendo más frágil a la albúmina ${ }^{(26)}$.

Tanto la calidad física como química del huevo son importantes, más aún cuando se incorporan nuevos ingredientes en la formulación en la dieta de las aves. A través de diversos análisis de laboratorio se pueden evaluar los cambios que sufren las muestras, tanto frescas, así como durante su almacenamiento, específicamente en las características físicas (peso del huevo y UH) y químicas (proteína, pH, aminoácidos y vitaminas), mismas que van a incidir en la calidad y preferencia por parte del consumidor. Según lo reportado por Grobas y Mateos ${ }^{(27)}$, los componentes hidrosolubles del huevo muestran escasa variación al modificar los ingredientes de la dieta del ave, sin embargo, estos autores no consideran los cambios debidos a las condiciones y tiempo de almacenamiento.

En la gallina, más significativo que la cantidad total de proteína, lo es el consumo diario de aminoácidos necesarios para producir la proteína del huevo. Los de importancia para el ave fueron cuantificados en HCC. Se han considerado como aminoácidos limitantes en las dietas para aves metionina, lisina y treonina, en HCC los contenidos de estos fueron suficientes, de tal forma que no fue necesario adicionarlos a la dieta, a diferencia de la testigo donde se añadieron para cubrir las necesidades del ave. Por lo tanto, la disminución de alguno de ellos afecta negativamente la producción de albúmina en el huevo, además de disminuir con el tiempo, el tamaño del huevo. La calidad de proteína está relacionada con la información genética de esta especie animal, misma que es considerada de un alto valor biológico, y como referencia para otro tipo de alimentos para consumo humano(28). De ahí la importancia que la dieta del ave lleve los niveles adecuados de los aminoácidos indispensables para mantener la calidad biológica y comercial del huevo.

La Norma Mexicana: Productos avícolas-huevo fresco de gallina-especificaciones de métodos de prueba $^{(7)}$, establece las características físicas y especificaciones que debe cumplir el "huevo fresco clasificado de gallina" que se produce o comercializa dentro del territorio nacional. En esta norma se reconocen cinco categorías en el huevo fresco de gallina, las cuales están determinadas por su peso y tamaño y se deben aplicar a todas las clasificaciones de consumo (extra grande= mayor a $64 \mathrm{~g}$; grande= 60-64 g; mediano = 55-60 g; chico $=50-55$ y canica menor a $50 \mathrm{~g})$. De acuerdo a las categorías 
anteriores, tanto el huevo testigo como con HCC el peso promedio del huevo fue de $63 \mathrm{~g}$ y al final (30 $\mathrm{d} / 20^{\circ}$ y $4^{\circ} \mathrm{C}$ ) fue de $60 \mathrm{~g}$, permaneciendo en la categoría de huevo grande.

Desrosier(28), observó que a los 15 días se presenta una pérdida de peso de huevo de 5 a $6 \%$ y a 30 días hasta de $10 \%$ sin importar el método de conservación. Otros autores reportan que a los 15 $\mathrm{d} / 7.2{ }^{\circ} \mathrm{C}$ se presenta una pérdida del $1.4 \%$, mientras que a $22{ }^{\circ} \mathrm{C}$ fue de $2.9 \%$, pero en refrigeración a 30 días fue del $3.1 \%$. Posiblemente los resultados reportados por estos autores y los de este estudio se deban a una pérdida de agua por evaporación, y esto es en función de la duración del almacenamiento, temperatura, superficie y porosidad del cascarón, así como la humedad relativa del medio ambiente. La evaporación es función lineal del tiempo de conservación y se traduce en una pérdida de peso y aumento en la cámara de aire ${ }^{(26,29)}$.

Así mismo, en la misma Norma Mexicana(7) se menciona la clasificación del huevo fresco de gallina para plato en los siguientes grados, conforme a las especificaciones: la escala de las unidades Haugh (UH) va de 0 a 110, y la interpretación de la misma es un auxiliar para determinar el tiempo de postura: a menor valor, mayor es el envejecimiento de éste. Por lo tanto esta clasificación será: México Extra mayor a $60 \mathrm{UH}$; México 1 de 61-70 UH; México 2 de 31-60 UH; Fuera de clasificación menor a $30 \mathrm{UH}$. En general, tanto en huevo testigo como el de HCC (76.1, 78.3 y $81.9 \mathrm{UH}$ a $20^{\circ} \mathrm{C}$ respectivamente) se clasificaron como huevo Extra grande $(>5.5 \mathrm{~mm}$ altura de albúmina y $>70 \mathrm{UH}$, para huevo recién puesto) disminuyendo para 15 y 30 días a $4{ }^{\circ} \mathrm{C}$, clasificándose como huevo Grande ( $>4.2 \mathrm{~mm}$ y 61 $70 \mathrm{UH}$ ) y a 30 días $/ 20^{\circ} \mathrm{C}$ como huevo Mediano (>3.0 $\mathrm{mm}$ y $31-60 \mathrm{UH})$.

En este estudio se presentó una disminución en las UH por efecto de la dieta, el tiempo y la temperatura de almacenamiento, siendo más notable en el huevo almacenado a 30 días $/ 20^{\circ} \mathrm{C}$. Esto se puede entender al presentarse una disminución de la calidad de la albúmina, reflejada en las UH, manifestándose por la licuefacción de la albúmina densa que tiene como consecuencia la pérdida de la estructura interna y de la organización espacial de las capas de la misma y de la yema ${ }^{(2)}$. Los mecanismos responsables de este cambio son complejos y no muy definidos, pero todos implican alteraciones de las proteínas como: 1) destrucción parcial del complejo lisozima-ovomucina debido a una inactivación de la lisozima; 2) destrucción de los enlaces electrostáticos entre los grupos amino de los residuos lisina de la lisozima y los grupos carboxilo del ácido siálico de la ovomucina cuando el $\mathrm{pH}$ se eleva; 3) disociación de las subunidades de la ovomucina, principalmente de las $\beta$, lo que se ve favorecido por el paso de iones divalentes desde la clara a la yema durante la conservación del huevo; 4) modificación de la estructura de la ovomucina, especialmente la subunidad $\beta$, lo que modifica el tipo de complejo lisozima-ovomucina, debido al aumento de $\mathrm{pH}$ y, 5) degradación parcial de los enlaces oglicosídicos, lo que produce la solubilización de la ovomucina- $\beta$, por la liberación de hexosas, hexosaminas y ácido siálico mediante un proceso de ß-eliminación alcalina(2).

Los fenómenos mencionados se relacionan con la liberación de anhídrido carbónico desde el interior del huevo, tendiendo a equilibrar su concentración con la tensión parcial con el aire circundante, dando como resultado un aumento en el pH. Las modificaciones se aceleran notablemente cuando el huevo se almacena a temperatura ambiente ${ }^{(29)}$. Lo anterior se presentó en los huevos almacenados a $20{ }^{\circ} \mathrm{C}$ por 15 y 30 días; al abrirlos se pudo observar que la yema se desplazó de su posición original (centro), se encogió, se aplanó y la membrana celular (vitelina) se rompió con facilidad. Todos estos cambios están asociados a las alteraciones generadas en las proteínas presentes en la albúmina.

Cabe destacar que aunque no hubo diferencias en la composición de las vitaminas hidrosolubles, cobra importancia cuando se considera el etiquetado de nutrimentos de los alimentos ${ }^{(30)}$.

\section{CONCLUSIONES E IMPLICACIONES}

De acuerdo a los resultados obtenidos en este trabajo se concluye la importancia que tienen la temperatura y el tiempo de almacenamiento en la conservación de la calidad de huevo, ya que se 
observaron los cambios físicos, químicos y mecánicos de los componentes internos del huevo desde la puesta hasta los 30 días de almacenado el huevo, siendo estos cambios irreversibles aun cuando las condiciones sean las óptimas. Se recomienda llevar a cabo este mismo ensayo con más tiempo de duración y llevar a cabo el análisis de digestibilidad de la proteína y aminoácidos de la harina de cabezas de camarón.

\section{LITERATURA CITADA}

1. Casas VM, Ponce D. Estudio del potencial pesquero y acuícola de Baja California Sur. SEMARNAP, Gob. Edo. BCS, FAO, INAPESCA, UABCS, CIB, CI CIMAR y CET del MAR. México, Vol. I. 1999.

2. Li-Chan EC, Powrie D, Nakai S. The chemistry of eggs and egg products. In: Yoshinori M editor. Egg bioscience and technology. New Jersey, USA: J ohn Wiley and Sons; 2008.

3. Goddard JS, Perret J SM. Co-drying fish silage for use in aquafeeds. Anim Feed Sci Tech 2005; 118:337-342.

4. Carranco J M, Calvo CC, Arellano ML, Pérez-Gil RF, Ávila GE, Fuente MB. Inclusión de la harina de cabezas de camarón Penaeus sp. en raciones para gallinas ponedoras. Efecto sobre la concentración de pigmento rojo en yema y calidad de huevo. Interciencia 2003;28:328-333.

5. Carranco J M, Sanginés G, Morales B, Carrillo D, Ávila G, Fuente M, et al. Shrimp head meal in laying hen rations and its effects on fresh and stored egg quality. Interciencia 2006;31:822-827.

6. UNA. Unión Nacional de Avicultores. 2015 [en línea]. http://www.una.org.mx Consultado 22 Feb, 2016.

7. NMX. Norma Mexicana FF-079-SCFI. Productos avícolas. Huevo fresco de gallina. Especificaciones y métodos de prueba. Secretaría de Economía. México. 2004.

8. Buba W, Dafwang II, Olugbemi TS, Opoola E, Iyiola-Yunji AO, Okafor EC. Effects of local storage methods on egg internal quality parameters during cold season. J Amin Prod Res 2013;25:45-51.

9. Ahn DU, Sell J, Chamruspollet M, Jeffrey M. Effect of dietary conjugated linolenic acid on the quality characteristics of chicken eggs during refrigerated storage. Poult Sci 1999; 78:922-928.

10. Guerra M. Factores que afectan la calidad del huevo. Agricultura 2000; (4):38-40.

11. AOAC. Official methods of analysis, Ass. Off. Agric. Chem. $17^{\text {th }}$ rev. ed. Washington DC, USA. 2000.

12. National Research Council, (NRC). Nutrient requirement of poultry. $9^{\text {th }}$ ed, Washington DC: Academy Press. Newsletter. 1994.

13. NOM. Norma Oficial Mexicana 062-ZOO. Especificaciones técnicas para la producción, cuidado y uso de los animales de laboratorio.
Secretaría de Agricultura, Ganadería, Desarrollo Rural, Pesca y Alimentación, México. 1999.

14. Charley $\mathrm{H}$. Tecnología de alimentos. Procesos químicos y físicos en la preparación de alimentos., México: Limusa; 2004.

15. Castro GM, Carrillo DS, Pérez-Gil RF, Calvo CC. Composición química de la langostilla y procesos tecnológicos. En: AureolesGamboa D, Balart EF editores. La langostilla: biología, ecología y aprovechamiento. Centro de Investigaciones Biológicas del Noroeste, S.C. México. 1995.

16. Cruz-Suárez LE, Ricque-Marie D, Martínez-Vega JA, Wesche-Eveling P. Evaluation of two shrimp by-product meals as protein sources in diets for Penaeus vannamei. Aquaculture 1993;115:53-62.

17. FAO. Joint FAO/WHO Codex Alimentarius Commission. Codex alimentarius. Food \& Agriculture Org. 1994

18. Andrade DR, Torres GR, Montes MEJ, Chávez BMM, Naar OV. Elaboración de un sazonador a base de harina de cabezas de camarón de cultivo (Penaeus sp). Rev Fac Química Farmacéutica, Colombia. 2007; 14: 109-113.

19. Toma RB, Meyers S. Isolation and chemical evaluation of protein from shrimp cannery effluent. J Agric Food Chem 1975; 23:632-635.

20. Quintero HE. Ingredientes para alimentos acuícolas - Parte I. American Soybean Association, 2009.

21. Huyghebaert G. Fisiología de la puesta con énfasis en la calidad de la cáscara. Selecciones avícolas. Abril 2006.

22. Berardinelli A, Ragni L, Giunchi A, Gradari P, Guarnieri A. Physicalmechanical modifications of eggs for food-processing during storage. Poult Sci 2008;87:2117-2125.

23. Estrada MM, Galeano LF, Herrera MR, Restrepo LF. Efecto de la temperatura y el volteo durante el almacenamiento sobre la calidad del huevo commercial. Rev Colomb Cienc Pecu 2010;23:183-190.

24. Zalapa RA. Correlación gráfica entre el peso del huevo, la altura de la clara y las Unidades Haugh. 2016. www.researchgate.net/publication/290523405. Consultado 20 Dic, 2016.

25. Scott TA, Silversides FG. The efect of storage and strain of hen on egg quality. Poult Sci 2000; 79:1725-1729.

26. Croguennec $T$, Nau F, Brulé G. Influence of $\mathrm{pH}$ and salts on egg white gelation. J Food Sci 2002;67:608-614.

27. Grobas S, Mateos G. Influencia de la nutrición sobre la composición nutricional del huevo. XII Curso de Especialización FEDNA. 1996.

28. Desrosier NW. Elementos de tecnología de alimentos. 10a reimpresión. México, DF: Compañía Editorial Continental SA; 1994.

29. Arias J L, Fernández MS, Nys Y. I mportancia de la calidad del huevo. 2007. www.tecnovet.uchile.cl/CDA/tecnovet_articulo.htm. Consultado 29 J un, 2015.

30. Naber EC, Squires M. Vitamin profiles of eggs as indicators of nutritional status in the laying hen: diet to egg transfer and commercial flock survey. Poult Sci 1993; 72:1046-1053. 